\title{
Research on Remote Monitoring and Control of Big Data Platform Based on Internet of Things
}

\author{
Jianmiao Zhu, Yunzhong Fang, Chenghang Jiang, Siyuan Hu
}

Zhejiang Academy of Safety Science and Technology, Hangzhou 310012, China

\section{基于物联网的危险源远程监控大数据平台研究}

\author{
朱建沗, 方云中, 蒋诚航, 胡斯源 \\ 浙江省安全生产科学研究院, 杭州 310012, 中国
}

\begin{abstract}
In order to give full play of big data on the safety production monitoring, To carry out the research on Hazard source safety monitoring service platform based on Internet of things. The platform based on the classification of major hazard sources, Safety parameter monitoring and control technology, Data standards for information collection and interaction, Management structure of supervisory system at all levels and Information sharing system framework. The platform has complete function, such as Basic information management, Dynamic monitoring, Big data analysis and Intelligent early warning. Constructing the supervision system of the four level linkage of the provincial city and county enterprise, collection and sharing of mass data of safety production, the law of accident happening rule can be summary by big data analysis model, realization monitoring and early warning by Man-machine collaboration and precision management of major hazard.
\end{abstract}

Keywords: Hazard source, Internet of things,b ig data, monitoring

\section{摘要}

为充分发挥安全生产监测监控大数据效应, 开 展了基于物联网的危险源远程监控大数据平 台研究。平台基于现有重大危险源分类分级、
安全参数监测监控技术、信息采集与交互的数 据标准、各级监管系统管理结构、信息共享系 统框架进行构建。平台具备基础信息管理、动 态监测、大数据分析及智能预警等功能, 构建 省一市一县一企业四级联动的监管体系, 对安 全生产海量数据进行采集与共享, 通过大数据 分析模型总结事故发生规律, 实现人机协同的 监控预警, 最终实现对重大危险源的精细化管 理。

关键词: 危险源; 物联网; 大数据; 监控

1. 引言

危险源对象是指工业生产过程中所需的 以及各种生产场所拥有的设施或设备, 如罐区、 库区、生产场所等对象。这些对象有各种易然、 易爆、毒性等危险物质, 对安全生产和人身安 全构成了极大的威胁。随着城市建设的发展, 一些有毒、有害、易燃、易爆等危险源有明显 向城市整体区域扩散的趋势, 由此产生的火灾、 爆炸和毒物泄漏等重大灾害事故也屡屡发生, 严重危及了公共安全, 因此必须把重大危险源 的控制与管理提到重要位置。

在事故预防以及危险源 “辨识-评价-分级 管理-监测监控-规划-应急” 技术体系中, “监 测” 占有至关重要的地位和作用, 同 “安全规 划” 等管理性手段相比, “监测监控” 虽然不 是处于事故控制的源头阶段, 但它是最接近实 际生产生活中的潜在事故控制对象, 因而其作 用最直接、效果更明显, 可以说 “危险源监测 
Risk Analysis and Crisis Response in Big Data Era (RAC-16)

监控” 是对评价、规划等安全管理技术的补充 和完善, 体现了 “关口前移、重心下移” 的安 全生产方针政策 ${ }^{[1]}$ 。目前重大危险源监测、监 控、预警技术，已成为我国当前安全科学领域 研究热点, 部分领域已经推广应用了一系列监 测预警系统，包括有限空间危险性气体检测分 析系统 ${ }^{[2]}$ 、重大危险源监控系统 ${ }^{[3]}$ 、尾矿库在 线监控检测系统 ${ }^{[4]}$ 等监测监控系统。但对于如 今的大数据时代, 上述研究成果还有待进一步 深入系统地集成与整合。

目前在重大危险源监测监控及预警技术 方面仍存在三大问题: 一是监测监控数据单 一、不成体系, 虽有部分数据联网, 但仍只是 信息孤岛; 二是现有监测监控监管以分散自 治为主, 没有建立省一市一县一企业多级联动 的监管体系, 缺乏相应的管理规则和制度。三 是监测监控技术的智能化有待提高, 安全生产 过程中产生的数据量大、类型多, 数据采集手 段有待优化和创新。基于以上分析, 本文把重 大危险源管理系统、在线动态监测系统、风险 评价系统集成到一起, 研究设计基于物联网的 危险源远程监控平台, 充分利用基于实时监测 的物联数据, 对危险源进行风险评级并分析事 故发生规律, 对事故进行预判预警, 有效预防 和减少安全事故。

\section{2. 系统设计}

利用物联网技术, 在可燃气体、危险化学 品、固体废弃物、危险设备等重大危险源领域 和其他高风险场所、装置的运行和管理状况进 行监测, 为监管部门提供在线即时监督监测, 实现非现场监督执法, 提高安全监管部门和相 关单位的安全生产日常监管、风险管理、预测 预警和应急处置能力, 有效降低安全生产事故 率, 并为掌控区域性安全生产总体情况提供服 务。同时平台也可以为企业提供预测预警服务, 并为企业的安全生产运行绩效留下可追溯的 电子台账。

2. 1 平台架构

基于物联网的危险源远程监控平台的设 计目标是为省一市一县一企业四级机构的安 全监管工作服务, 实现分级管理, 提高监管效 率, 保障安全生产。按照分级监控与管理的原 则, 设计了四级监控预警中心 ${ }^{[5]}$ 。系统监管体 系组织结构如图1所示。

\section{2 平台功能定位}

平台主要分政府层面和企业两个层面进 行设计, 从政府角度分析主要承担为各级安监 部门全面、准确、快捷掌握生产企业的实时生 产状况, 了解各类重大危险源详细的生产状况

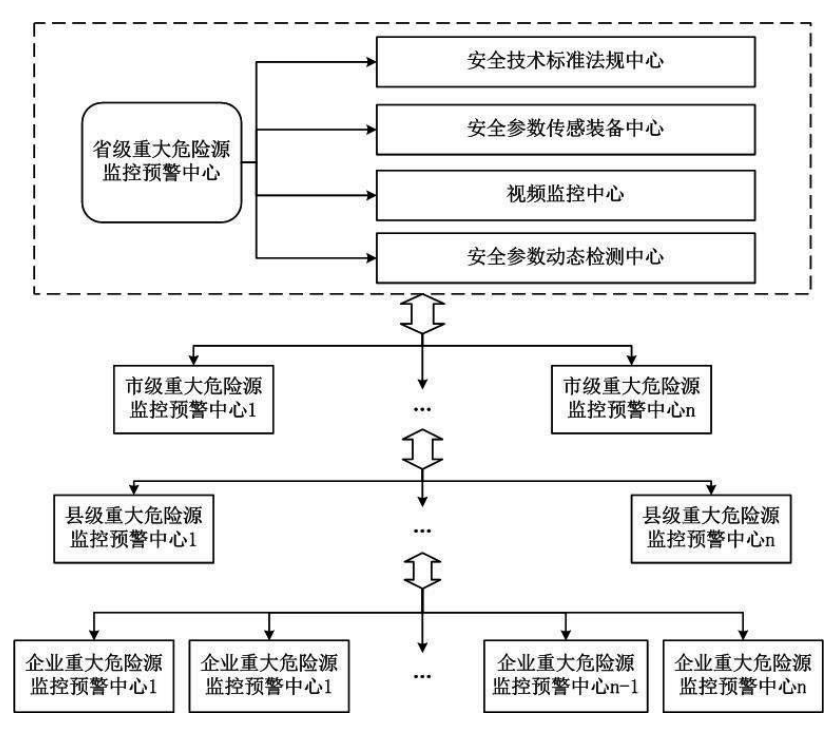

图 1. 危险源远程监控平台组织结构体系 
Risk Analysis and Crisis Response in Big Data Era (RAC-16)

及位置分布; 帮助监管部门全面掌握所监管辖 区内生产企业的安全生产情况, 如各企业的基 本情况、各企业重大危险源的情况、应急预案、 生产过程中的规范要求及安全人员的监督状 况等，实现企业的安全监管。同时通过实时监 测积累辖区重大危险源安全生产运行数据, 利 用安全生产分析模型进行大数据分析, 形成辖 区安全生产分析报告 ${ }^{[5]}$ 。

从企业角度分析则实现对各类涉及安全 生产车间、仓库等场所的重要数据 (如有毒气 体浓度、温度、压力) 进行监测, 超限时进行 报警, 并通过数据分析对相关区域安全状况进 行评定等。通过网络, 如计算机、手机, 企业 管理人员不管身在何处, 可以实现对本企业重 点部门的实时现场监控, 并可发出相关指令。

\section{3. 系统架构}

3. 1 系统总体框架

系统总体框架见图 2 。

\section{2 危险源监控网络接入}

危险源监控的主要工作是实现企业现场 各种设备串口（包括RS232、RS485、RS422 等)、以太网、各种现场总线 (包括 CAN、 LonWorks、Profibus等) 通讯网络的相互转换, 以便与其它设备或调度间进行实时的数据采 集、传输和交互。因此企业端监控端须集成通 信采集规约库和转发规约, 可以实现采集多个 不同子系统的数据, 并进行数据集中汇总、分 类和预处理, 对上级数据中心进行数据转发, 实现企业端和政府端的有效链接。

3.3 危险源数据中心

数据中心管理平台是积累大数据的重要 组成部分, 包括实时数据库和关系数据库。其 中实时数据库是监测的基础数据平台, 需具有 高性能、高吞吐能力、可靠性强和跨平台的功 能, 具备海量的数据存储能力, 单节点支持百 万级测点, 实时收集、获取企业运行过程中的 各种监测数据, 并将其转化为对各类业务有效 的公共信息, 将危险源分布广的特点做集中管

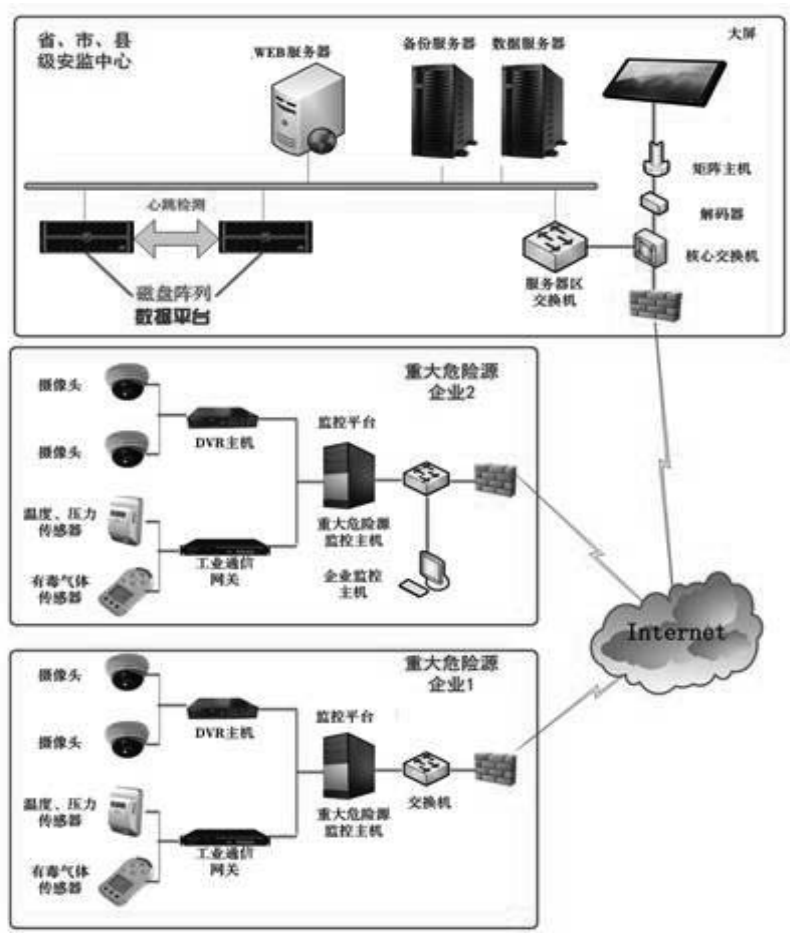

图 2. 危险源远程监控平台系统总体框架 
Risk Analysis and Crisis Response in Big Data Era (RAC-16)

理。通过关系数据库的融合应用, 实现企业安 全生产管理、过程监控、风险评估以及政府监 管之间对实时信息完整性、一致性、安全共享 的需求, 可为企业与政府 2 个不同的系统建立 起信息沟通的桥梁。

\section{4. 企业端系统功能}

企业端系统主要完成对各类涉及安全生 产车间、存储区等场所的实时参数 (如有毒气
体浓度、温度、压力、液位）进行监测, 超限 时进行报警。并通过数据分析对罐区安全状况 进行评定等。通过网络, 如计算机、手机, 使 企业管理人员不管身在何处，可以实现对本企 业重点部门的实时现场监控, 并可发出相关指 令。

4.1 设备实时监控

系统可以实时监控设备状态, 以及对相关 参数进行设置 (图3)。

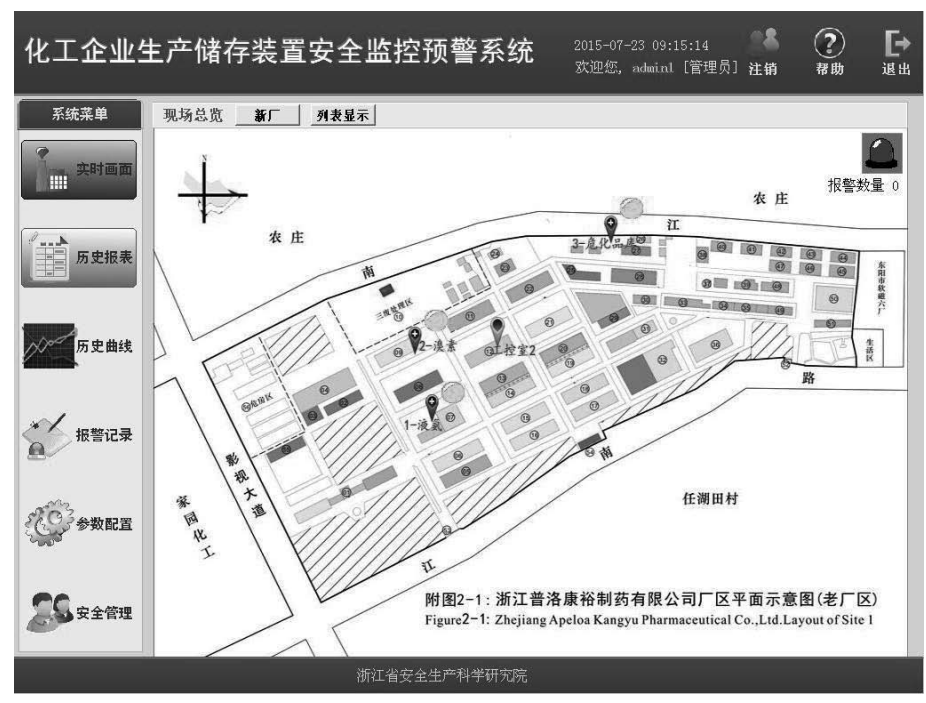

$\oplus$

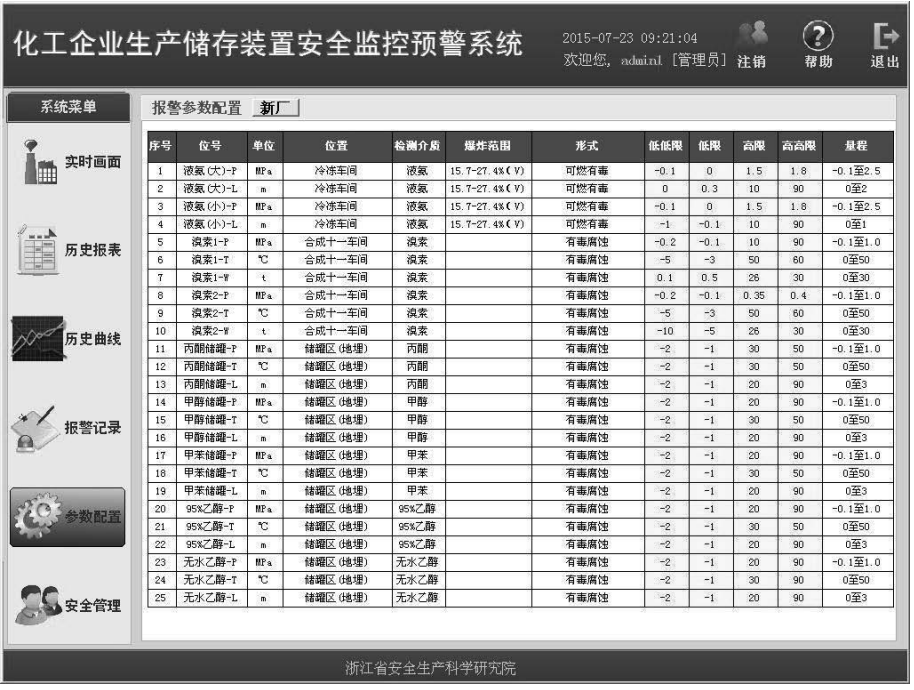

图 3. 设备参数设置 


\section{Risk Analysis and Crisis Response in Big Data Era (RAC-16)}

\section{2 报警管理}

系统可实时显示并记录现场危险源参数 的各种报警信息, 同时可将报警内容通过邮件、 短信等方式发送给对应的人员。

4.3 趋势曲线和报表

提供查询任意变量的实时历史曲线, 以及 任意时间段的历史数据报表（图4）。
利用安全生产分析模型进行大数据分析, 从海 量的重大危险源安全生产监测监控的数据中 寻找安全事故发生的规律及特征, 对重大危险 源的准确预警, 并形成辖区安全生产分析报告, 提高安全生产监管效率。

5.1 大数据分析效应

大数据是指具有 “大容量 ( volume)、多

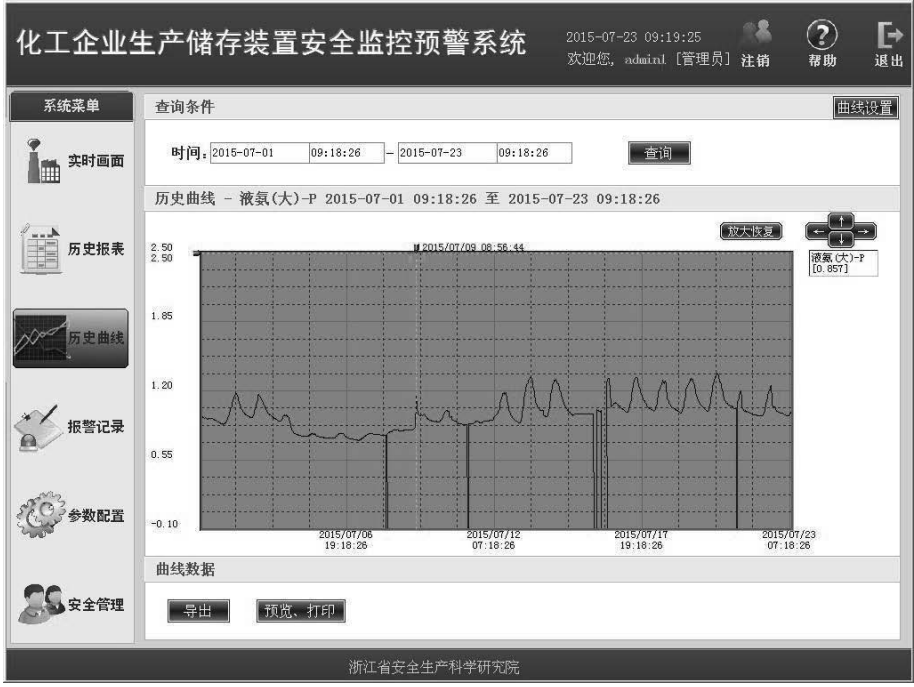

图 4. 趋势曲线和报表

\section{4 日志查询}

将各种组件的状态信息和相关通信信息 统一管理起来, 用户可以通过日志来了解软件 的运行情况以及操作记录。

4.5 与关系数据库的交互

监控中心实时监测数据库与关系数据数 据库之间的交互, 即将监控中心的实时监测数 据进行统计并转存到关系数据库中, 并为上层 分析功能应用提供了一个新的查询历史数据 入口, 经过统计后的数据能更方便的被上层应 用所用。

\section{5. 政府端系统功能}

政府端系统功能主要是获取企业端监测 数据, 了解各类重大危险源详细的生产状况及 位置分布。积累形成大数据, 构建重大危险源 动态智能监测监控大数据分析平台, 并在此基 础上改善重大危险源监测监控预警方案, 提出 辅助安全生产监管工作科学决策的分析模型,
变性( variety)、快速化( velocity)”特性的数据。 随着安全生产监测监控系统的开放与应用, 各 种安全生产重大危险源的安全参数大数据正 以惊人的速度呈爆炸式地增长, 而企业级的重 大危险源安全生产监测监控系统所监测和建 立的数据库仍是小型的、单一的、孤立的, 基 于局部范围的抽样样本统计, 各监测监控系统 的数据相互对立没有形成对接共享。

若要发挥大数据效应则要求穷尽一切相 关样本, 尽可能搜集全面, 不是拥有支离破碎 的割裂数据, 而是完整的数据。数据的海量及 其完整性比传统数据库具有相当的信息优势。 构建重大危险源动态智能监测监控大数据分 析平台, 可以整合各业务系统数据, 并在此基 础上改善重大危险源监测监控方案, 提出辅助 安全生产监管工作科学决策的分析模型 ${ }^{[6]}$, 通 过数据生成、数据采集、数据储存以及数据分 析等 4 个阶段, 形成应用安全生产重大危险源 
Risk Analysis and Crisis Response in Big Data Era (RAC-16)

大数据, 从中寻找安全事故发生的规律及特征, 实现对重大危险源的准确预警, 对症下药, 有 效遏制事故的发生。

5.2 政府端平台智能监管技术分析

通过企业端的安全参数监测传感器采集 重大危险源的相关信息传输至危险源远程监 控大数据平台, 利用重大危险源动态预警模型, 通过大数据分析平台, 比对分析其他相关数据, 实时评价重大危险源的生产状态和安全状态, 根据安全评价结果输出预警报警信号, 给出报 警分析、事故分析、操作指导及改进建议等信 息。
采集规约库和转发规约, 可以实现采集多个不 同通讯模式的实时数据, 并进行数据集中汇总、 分类和预处理, 对上级数据中心进行数据转发。

(4)标准化体系: 为实现信息平台数据交换 和共享、避免数据库成为 “信息孤岛”，从分 类编码、元数据到数据更新维护形成系统的一 套标准体系。

6.2 实际应用

(1) 浙江普洛康裕制药有限公司是一家集 研究、开发生产原料药、制剂及医药中间体的 综合性制药企业, 生产过程中涉及众多危险化 学品, 如, 液氨、溴素、甲醇等危化品存量较

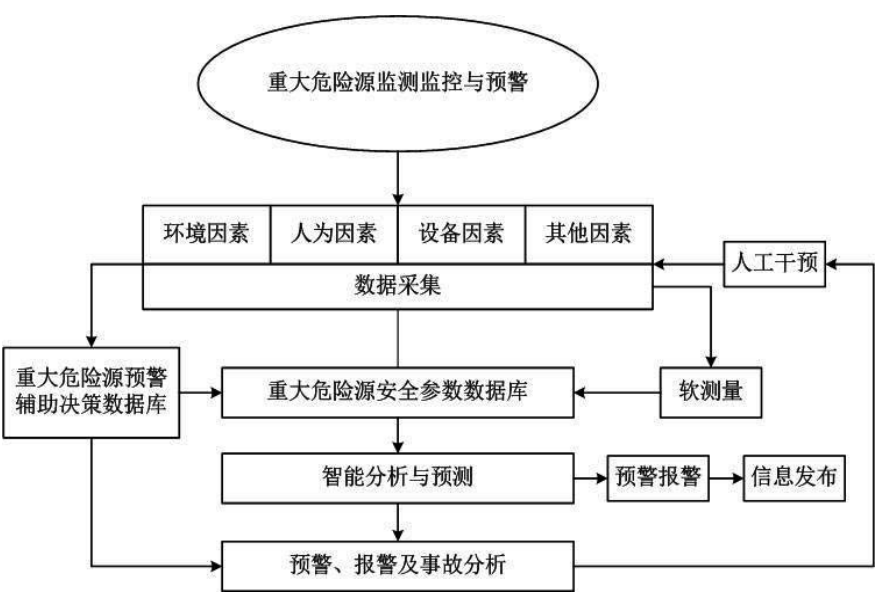

图 5. 危险源远程监控平台监测监管及预警流程图

危险源远程监控平台监测监管及预警流 程如图5所示。

\section{6. 平台技术特点和实际应用}

6. 1 技术特点

(1)信息平台数据库系统通过实时数据引 擎技术和压缩技术, 实现了实时监测数据的有 效保存和高效读取, 同时通过关系数据库的设 计实现了监测数据、管理信息、多媒体和图片 数据统一存储。

(2)通过危险源数据中心建设, 构建了三 层服务结构, 政府端不直接访问企业端数据库, 而是通过中间服务层与数据库服务进行交互, 提高了数据服务的安全和效率。

(3)企业端监测数据交换技术，集成通信
大, 构成了危险化学品重大危险源, 其原有的 储存安全监测数据不完整, 不具备数据远传功 能, 基于上述情况, 对其进行了企业端监测系 统的升级改造, 实现对重大危险源监测传感器 的补充完善和数字化改造, 实现了安全监控预 警参数（压力、温度、液位、质量）的集中监 控和数据远传。经过半年多的运行, 系统运行 稳定, 实现了普洛康裕公司危险源全天候监测, 达到了企业的安全管理目标和政府部门对危 险源管理的要求。

(2) 目前浙江省安全生产科学研究院正着 力打造省一市一县一企业多级联动的安全生 产监管体系, 已在浙江省杭州市建设省级安全 生产监控中心, 定位于为省市两级政府部门提 
Risk Analysis and Crisis Response in Big Data Era (RAC-16)

供安全生产大数据分析; 在浙江省湖州市吴兴 区建设区县级监控服务中心, 定位于安全监测 数据监控并根据监测数据为企业提供技术服 务和隐患排查服务。目前政府端系统已经开始 接入浙江省危化品登记数据以及重大危险源 实时监测数据, 后续将持续接入有限空间危险 性气体环境监测数据、用电安全监测数据等, 为浙江省安全生产管理提供数据支撑。

\section{7. 结语}

安全生产水平是衡量一个国家现代文明 程度的重要指标。近年来, 我国安全生产形势 总体趋于稳定, 表现在事故总量下降, 工矿商 贸行业安全生产状况趋于好转, 建筑、交通、 烟花爆竹和民爆器材等相关行业和领域的安 全工作取得成效; 但另一方面安全生产形势依 然十分严峻, 与欧盟、美国和日本等发达国家 相比, 我国生产事故总量较大, 重特大事故频 繁发生, 职业危害严重 ${ }^{[7]}$ 。随着信息化的发展, 互联网和物联网技术的应用越来越广泛, 因此 在安全领域中充分利用信息化技术提升本质 安全尤显重要。

基于物联网的危险源远程监控平台采用 动静结合的数据库模式, 可为判定危险源的安 全状态提供更为全面、可靠的依据。平台实现 了对安全生产数据的采集与共享, 有效发挥安 全生产过程中监测监控参数的大数据效应, 通 过海量数据的整理分析, 总结事故发生规律, 提高安全生产事故预警精度 ${ }^{[8]}$, 提升安全生产 监管水平, 为安全监管提供技术支持, 实现对 重大危险源的精细化管理, 但大数据的发掘与 分析模型、预警模型仍需进一步研究与优化。

\section{Acknowledgements}

This study was supported by Science and technology project of Zhejiang Province (No.2016F10028\&No.2014F50024) \& Science and technology project of Hangzhou (20150533
B57)

\section{致谢}

本研究得到了浙江省科技计划项目 (2016F10028、2014F50024)和杭州市科技局项 目(20150533B57)的资助。

\section{参考文献}

[1] 吴宗之. 论城市重大危险源监控与应急救 援体系建设. 安全,2005,02:6-8+5.

[2] 黄文宏, 李学盛, 王海龙, 陈兵. 典型石化有限 空间危险气体在线监控系统构建.浙江化工, 2011, 42(6):25-27.

[3] 蒋诚航,李学盛. 基于组态系统的重大危险 源储罐监控系统研究. 浙江工业大学.安全 生产应急管理与安全信息化的实践研究 -2015 浙江. 第四届安全科学与工程技术 研讨会论文集.浙江工业大学,2015:5.

[4] 杨杰, 李青. 地下位移实时监测系统研究, 中国计量学院学报,2008,01:41-46.

[5] 朱建沝,汪圣华,张金峰. 基于物联网的安 全生产隐患实时监控服务平台的构建. 浙 江工业大学.安全生产应急管理与安全信息 化的实践研究一2015 浙江·第四届安全科 学与工程技术研讨会论文集. 浙江工业大 学,2015:5.

[6] R. Bubbico, Efficient applications of risk analysis in the chemical industry and emergency response, Journal of Risk Analysis and Crisis Response, 2011, 1(2): 92-101.

[7] Z.Z. Wu, S.Z. Zhang, M.R. Zeng. Analytical research on the accident risk in three industries of China and USA in the period of 2006-2010. Journal of Risk Analysis and Crisis Response, 2013, 3(1):52-58.

[8] 曾胜. 重大危险源动态智能监测监控大数 据平台框架设计. 中国安全科学学报, 2014,11:166-171. 